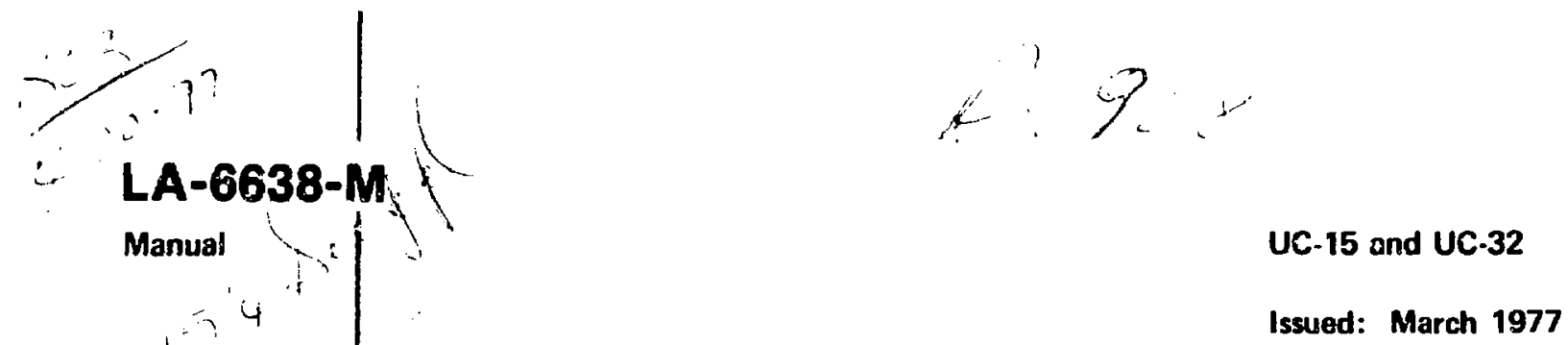

\title{
Effluent and Sanitary Sewer Monitors
}

\author{
by
}

L. Stanchi*

M. R. Vasey *

"Visiting Staff Member. Euratom Joint Research Center, Ispra, Italy.

*"Missouri Research Laboratories, Albuquerque, New Mexico 87102.

\section{scjentilic laboratory}

of the University of California

LOS ALAMOS, NEW MEXICO 87545

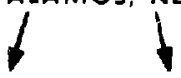

An Alfirmative Action / Equal Opporlunity Employer 
Printec in the United States of America. Available from National Technical Information Service

U.S. Department of Commerce

5285 Port Royal Rond

Springfield, VA 22161

Price: Printed Copy \$4.00 Microfiche $\$ 3.00$

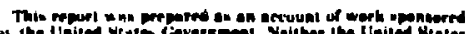

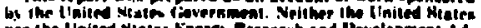

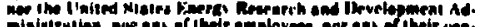

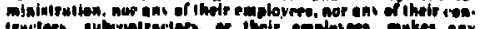

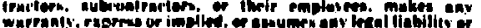

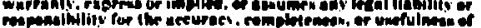

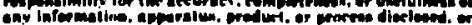

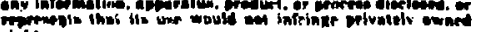
rithise. 


\title{
EFFLUENT AND SANITARY SEWER MONITORS
}

\author{
by
}

L. Stanchi and M. R. Vasey

\begin{abstract}
Two similar instruments that monitor the liquid wastes from the plutonlum facility are described. The operation of the two instruments is completely automatic and periorms a continuous surveillance in the frame of Nuclear Safeguards. One instrument controls the liquids from the facility and the other checks the sanitary sewer wasteg. Both have self-diagnosing capabilities and take autamatle actions in case of abnormal occurrences.
\end{abstract}

\section{INTRODUCTION}

This manual deals with two similar instrumente that monitor the effluents from the plutonium facility to prevent environmental pollution with radioactive waste and also to safeguard strategic nuclear material by avoiding its fraudulent diversion.

The first instrument, Effluent Monitor System, is the more complex; it is related to two analog chains for acquiring data from low- and medium-energy particles. The second instrument, Sanitary Sewer Monitor System, looks at low-energy particles only.

The electronic circuitry is practically the same for both instruments, the second being obtained from the first by eliminating unnecessary parts with few minor modifications.

The programs are also similar but they are arranged differently. A general description of the first instrument' indicates the reasons for the choice of the detectors and the hardware. The second instrument uses the same concepts and will not be described in detail here. However, full details about hardware, software, and operative procedures are outlined in the following sections.

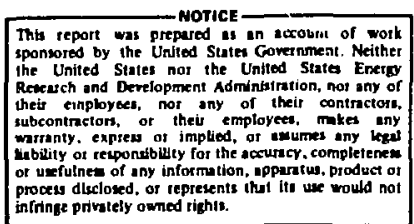

The general description in Ref. 1 should be referred to even though additions and minor modifications have been made.

\section{OPERATIVE PROCEDURES}

Both instruments stand alone; they operate continuously without surveillance by the operator.

Both instruments can acquire data about radioactivity in the effluent and can take action if a preset threshold is exceeded. Both also have selfdiagnosing capabilities for correct operation.

The operator has access to three switches and two pushbuttons in addition to the keyboard. The switches for Power-on, Automatic Power Restart Enable (APRE), and Audible Warning Enable (AWE) are reached by opening the front panel. The pushbuttons on the front panel stop the operation or reset the alarms. Other features can be called by the operator via the keyboard. If a key is pressed, the program answers by calling the requested procedure or by printing either WRONG INITIAL or ILLEGAL CHARACTER, depending on the first character that was typed. The legal initials are C, E,

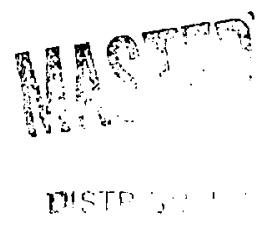


I, $O, P, S$, and $T$, corresponding to the available procedures CONTINUE, EXPER, INITIALIZE, OPER, PRINT, START, and TEST. ILLEGAL COMMAND is printed if the operator attempts to interrupt the calibration sequence.

The instrument starts automatically if APRE $=1$. The first time the power switch is set to $O N$, the content of the operative constants is random and it must be initialized. If the operator switches the power OFF and ON, the value of the constants stored in C-MOS RAM will not disappear and can be recovered.

When energized the instrument goes to warm up if $A P R E=1$ or to a standby position (EXIT $=H A L \Gamma$ ) if $\mathrm{APRE}=\boldsymbol{\phi}$.

The operator can call one of the listed procedures that causes a program incerrupt by typing the desired name, e.g., INITIALIZE or START.

INITIALIZE allows the operator to set the values of the operative constants. Twelve messages are automatically displayed on the teletype printout for setting the values of time and number of measurements, the Alarm 1 threshold, the limits for source counting, and the values of the corrective constants for peak evaluation. The operator types an $X$ plus carriage return (CR) if the displayed number in any particular message is correct. If it is not correct, the operator types the desired number plus carriage return, and the new number is stored in C-MOS RAM, replacirig the old one. Then the next messege is displayed, to the end of the twelve messages. END OF INITIALIZE is then printed followed by a prompt character (?) requesting new instructions. At this point, the operator normally will begin the operation with the START or CONTINUE com. mand.

START forces the instrument to start with a 2 . min calibration sequence composed of three phases. The three pheses are (1) MOVI (30 s), which brings the sources into the calibrating position; (2) STABILIZE $(60 \mathrm{~B})$, which allows for source counting (Now, the analog stabilizers are activated to compensate for drifts in the detector and the analog chain.); and (3) MOV2 (30 s) which brings the sources back to the rest positious. Note that ir is illegal to interrupi the calibration sequence; if the operator presses any key + CR during this 2-min time, the command is refuted and ILLEGAL COM.
MAND is printed. The only exception is PRINT. which is accepted at any time.

CONTINUE allows the operator to skip the calibration and to go directly to measurements. Normally this is done when a calibration has just been completed and the operator wishes to change. for example, the alarm threshold, and then restart immediately. In this case a new calibration is pleonestic and CONTINUE allows an immediate measurement.

EXPER is used to test the hardware with a recurrent signal thet can be checked easily with the oscilloscope. This procedure sends ones and zeros alternately with one of six different infinite loops. It was reduced for the EFFLU program because it exceeded the 4096 bytes allowed by the PROMs assembled on the single-board computer. It has been used with the Effluent Monitor by neglecting ALARM 4. The SEWEFF program has this prccedure as an integral part.

OPER $^{2}$ is used to store an auxiliary program in RAM for future use. The operator types codes in machine language terminated by a slash $(/)$. The auxiliary program then is executed ustomatically. See Ref 2 for details. Some difficulties due to the ASCII codes are outlined in Ref. 3.

PRINT gets a flag that is used by the program at the end of the measurement to print data corresponding to corrected peak areas, or at the end of the stabilization to print the source counts. The number of successive neasurements printed is governed by a number stored in RAM during in. itialization. This procedure interrupts the calibra. tion sequence to print the source contents. The PRINT flag is reset only at the beginusing of each measuremeni and successive measurement results can be printed if the operator has fixed the number sif prints at greater than 1. If the operator sets the PRINT flag during the last measurement before calibration there is the double effect of printing the results of that measurement plus the source contents at the end of the successive calibration.

TFST is used by the operator to gate off the connection to the building computer so that he can test conditions that provoke alarms, without sending unwanted warnings. The TEST condition can be quite dangerous if the operator forgets to reset the TEST flip-flop. To prevent this cccurrence, it is reset 
automatically, as is the PRINT flag, at the beginning of each measurement. The TEST flag must be set before entering the calibration sequence if it has to block Alarm 2 conditions.

The above procedures set the values of particular bytes called operative fields, which have well. defined addresses.

Addresses of the instrument are given in Table I, whereas bit assignments of the OP fields are shown in Table II.

\section{FRONT PANEL INDICATIONS}

Figure 1 shows the completed instrument with details of the front panel. The POWER, APRE, and AWE switches are located behind the front panel and are not accessible without opening it. Two manual commands are available on the front panel: the STOP pushbutton, which stops the operation and disables the hardware, and the MANUAL ALARM RESET, which is useful in preliminary operations.

Two vertical rows of red LED lamps indicate the operative phases and status, respectively. The names near the lamps are self-explanatory. The ENABLE status of the hardware is indicated by two lamps switched by the $30-8$ clock that controls the entire operation. The two lamps also provide the clock time.

The phase lamps indicate the succession of operative phases. The warm-up phase occurs at the beginning and the others follow with cyclic permutation. A preestablished number of measurements is performed before each calibration, and the operator can determine the time of each measure- ment as well as the number of measurements in a set by the switching of the double lamp indicating the measurement phases.

Two blue lamps indicate $A P R E=1$ and $A W E=1$. They warn, when lit, that automatic power restart and audible warnings, respectively, are enabled.

Five lamps indicate the alarm conditions. A very impressive flashing yellow lamp indicates Alarm 1, three red LED lamps indicate the group of Alarm 2, and a green lamp indicates the presence of power. (Lack of power will darken this lamp and set Alarm 3.) Alarms 4 and 5 use a combination of lamps, as described below.

\section{ALARMS}

Different alarms warn the operator when something performs incorrectly during each phase. In these occurrersces, actions will be initiated automatically. The various alarms with their consequent actions are listed below.

Alarm 1 is set if the $17-\mathrm{keV}$ counts in the selected time exceed the predetermined numerical hreshold. Both time and threshold are stored in CMOS memory. If Alarm 1 is set, the building computer is warned, and local actions are taken. The nlashing yellow lamp goes on and a double audible warning-local and remote-starts. (Audible devices can be silenced by the operator.) At the same time data corresponding to the $17-\mathrm{keV}$ counts are sent to a chart recorder via a digital-to-analog converter (DAC), and the recorder is started. The recorder uses a thermal pen because a normally idle instrument cannot use an inked pen safely. The contents

TABLE I

MEMORY ADDRESSES

\begin{tabular}{l}
\multicolumn{1}{c}{ ITEM } \\
\hline On-board PROMs \\
On-board RAMs \\
C-MOS RAM \\
Counters and OP \\
fields
\end{tabular}

HEX ADDR.

0000 - OFFF

3CO0 - 3FFF

4000 - 4 OFF

$8000-8007$
DECIMAL ADDR.

$0 \quad-4095$

$15360 \cdot 16383$

$16384-16639$

$32768-32775$ 
TABLE II

BIT ASSIGNMENT

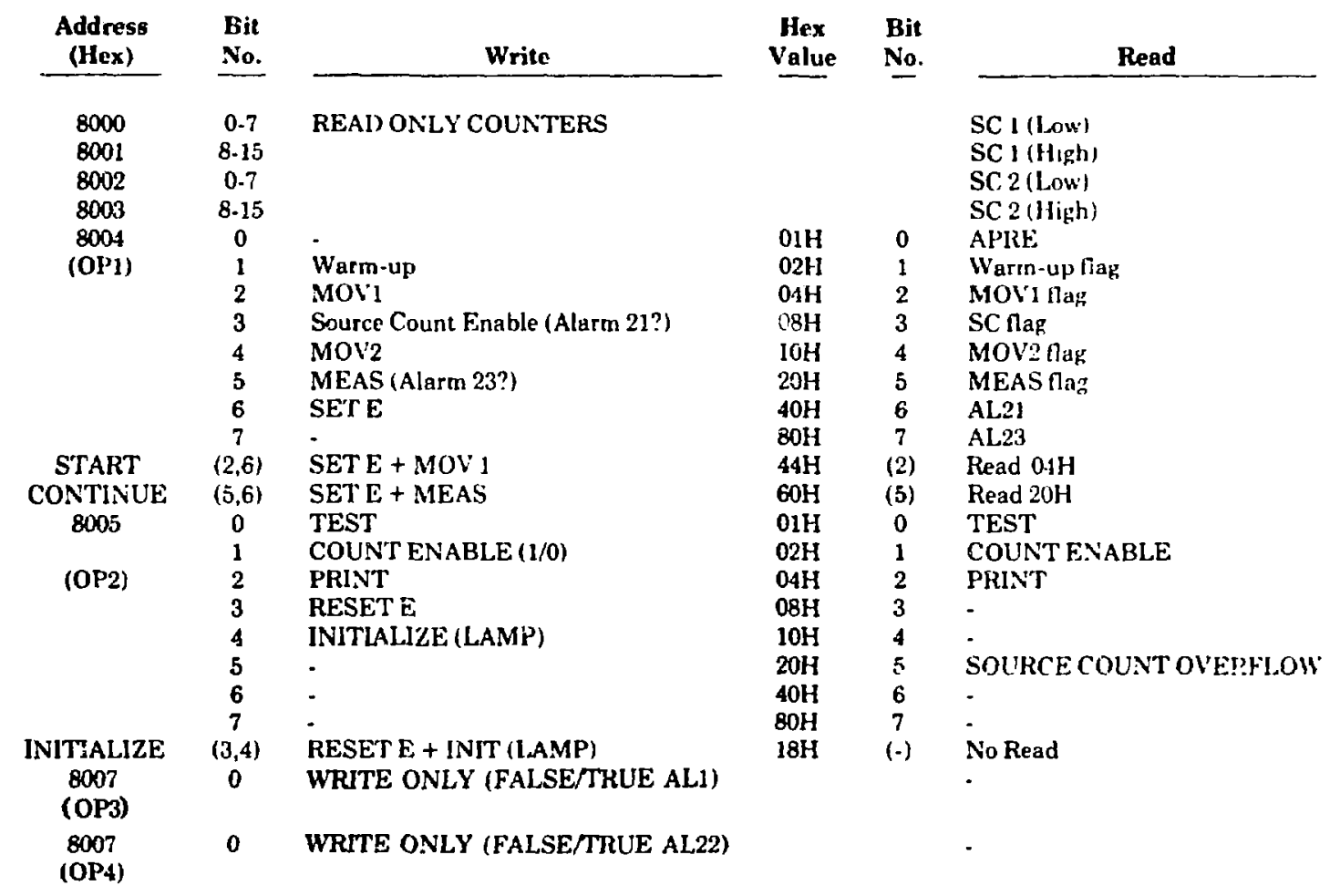

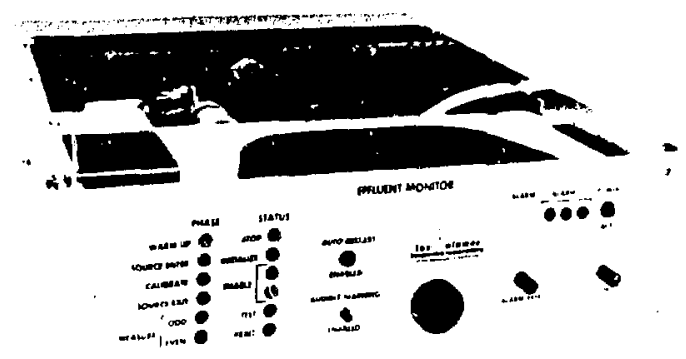

Fig. 1.

Front Panel of the Monitor. The same port panel is used by Effluent and Sanitary Sewer Monitors. of the three peak areas $(17 \mathrm{keV}, 59.6 \mathrm{keV}$, and 400 $\mathrm{keV}$ ) are corrected with the equation

$\mathrm{A}=\mathrm{P} \cdot \mathrm{kB}$,

where $\mathrm{P}$ is the content of counts between the $\mathrm{am}$ plitude threshclds that select a peak in one of the single-channel arialyzers (SCA) and B is the total count in the background, corrected with the constant $k$ preset by the operator and stored in C-MOS RAM.

The difference can be negative if the constant is not chosen properly or if the number of pulses is so low that statistica! fluctuations reverse the normal situation. As the peak of $17 \mathrm{keV}$ sets Alarm 1 if the threshold is exceeded, a suitable constant is added. The evaluation for the $17-\mathrm{keV}$ area is thus

$$
A=\operatorname{Corr}+\mathrm{P}-\mathrm{kB},
$$


where the constant Corr also is stored (as STOCORR) in C-MOS RAM. It is quite clear that a small negative number is seen as a large content given by the four hexadecimal digits allowed for counting. As an example, -1 is seen as FFFFH, which in decimal is 65535. The occurrence time of the alarm is not printed because it results in a duplication of the real-time counting of the building computer and it becomes meaningless after a power failure.

Last, but not least, Alarm 1 drives the holding tank controller. This is now only a provision that the hardware is performing. When the holding tank and its controller have been constructed, the effluen $i$ will be diverted automatically to the holdint tank, from the first occurrence of the alerm until the end of the first nonalarmed measurement.

Alarm 2 is set up during calibration and is, in effect, a group of three alarms that take different actions. Alarm 21 is related to MOV1. If the source position is not confirmed by an optical sensor, the action is to stop the instrument completely and warn the building computer. No recovery is possible without intervention by the operator. Alarm 22 is related to source counting. If one or both source counters are outside their own high or low limits (stored in C-MOS memory), the source counts are printed automatically and a warning is sent to the building computer. The inst:ument is not stopped, but there is no guarantee that the analog chain will perform correctly. Alarm 23 is related to MOV2 and is similar to Alarm 21.

Alarm 3 is established by the lack of power and disables all the hardware in the instrument before the voltage is completely down, taking only the external action of warning the building computer by opening a normally closed (N.C.) connection.

Alarm 4 is very particular and is related to the lack of pulses in any one of the five pulse counters. This lack can be caused by a hardware inperfection and is software implemented only. The imperfection can be a bad contact, a broken wire, or a noninserted coaxial cable. The lack of pulses can also occur because of a failure in the detectors or in the analog chain, both in the amplification part or in one single-channel analyzer (SCA). If one of the counters receives no pulses, Alarm 4 is set. During correct operation it is impossible to have no counts, therefore a perfect zero is reason for alarm, es- pecially because a lack of pulses in the $17-\mathrm{keV}$ counter will not set Alarm 1, which is related to the main abnormal condition that the instrument has to evidence. This could be a way to tamper if a suitable diagnosis is not made. When Alarm 4 is set, a coding from Alarm 2 group is performed. Alarm 4 is indicated by the simultaneous presence of Alarm 21 + Alarm 23, which is impossible in the normal succession of phases.

Note that Alarm 4 raises the PIINT flag so that peak areas are displayed. A zero is seen in the area or areas corresponding to the incoming zero. The numbers, together with AL21 + AL23, will be quite clear to the operator. If the three areas show normal counts, this means that a zero was in one of the background counts not displayed.

It seems useful not to stop the operation because, even in this condition, other actions can be perfor. med by the instruments before the intervention of the orerator. For example, if Alarm 4 is given by a counter other than the $17 \mathrm{keV}$, Alarm 1 can be recognized afterwards and the necessary action taken.

Alarm 5 takes care of a lack in the clock interrupt circuitry. Unlike Alarm 4, this is only hardware implemented. During the operation, a contact related to the dialog between CPU and hardware can fail. Three contacts on the 100-pin connector to the hardware board can cause a clock interrupt failure. and these have been reinforced by putting a duuble co.itact on both sides of the connector. The failure is now less probable, but a suitable warning is necessary. Assuming that the operator relies on the instrument, which normally behaves regularly, he gives it perhaps only a quick glance every day. He arrives, everything seems all right, and the ENABLE lamps are switching regularly every $30 \mathrm{~s}$. But if the interrupt due to clock has not been received by the CPU, the MEAS lamp is not switched. We cannot rely on the operat ar waiting maybe $10 \mathrm{~min}$ (if this is the selected measurement time) to see if the MEAS lamp switches. Assuming that the interrupt is not received by the CPU, no action is taken by the instrument, which becomes useless if no suitable diagnostics have been provided. For that purpose an appropriate diagnosis capability is provided by the hardware. A time-out failure is related to the clock, setting up the ALARM 5 flip-flop by the hardshaking feature of 
the Programmable Peripheral Interfaces (PPI) mounted on the single-board compuier. The interrupt is set by the pulse $\overline{\mathrm{STB}}_{\mathfrak{y}}=\overline{\mathrm{CLST}}$, which provokes feedback of the $\overline{\mathrm{IBF}}_{\mathrm{B}}$ pulse. This pulse resets flip-flop U2-6A. If either pulse is lost, the flipflop is not reset, and Alarm 5 is sent to the building computer. This is encoded as AL3 together with AL22, so it can easily be recognized.

No interrupt is established if $\mathrm{IBF}_{B}$ is lacking, so only a hardware operation can be effective.

Alarm 6 is a minor alarm. If strobe $\mathrm{STB}_{\mathrm{a}}$ is present but nothing is received by the CPU, a software correction is made. $\mathrm{STB}_{\mathrm{B}}$ can be operated only by a clock or a stop. In case of a stop the operator is present and can immediately ascertain whether the significant bit is lost. If the bit indicating a clock is lost, this is considered the situation for folarm 6 . As the strobe $\mathrm{STB}_{\mathrm{B}}$ cannot arrive without a clock, the program instructs the $\mathrm{CPU}$ to behave as if a clock has been received. But to show the difference from normal operation, NO CLOCK is printed.

Table III shows the alarms with their automatic actions.

TABLE III

\section{ALARMS AND THEIR ACTIONS}

\section{Alarm Type and Cause}

ALARM 1

Excessive radioactivity

ALARM 21

MOV1 not confirmed

ALARM 22

Calibration not OK

ALARM 23

MOV2 not confirmed

ALARM 3

Power off

\section{ALARM 4}

Pulse counter $=0$

\section{ALAKM 5}

CLST strobe fails
ALARM 6
Clock bit not
received

Action

ALl to B. C $^{-}$

Prints data

Diverts effluent

AL21 to B.C.

Stops operation

AL22 to B.C.

Prints source data

AL23 to B.C.

Stops operation

AL3 to B.C. Operation stopped

AL21 + AL24

to B.C.

Prints data

AL3 + AL22
to B.C.

Prints message

NO CLOCK
Lamps

Flashing

yellow AL1

Red AL21

Red AL22

Red AL23

Green AL3

off

Red AL21

Red AL23

Red AI,22

Green AL 3 off

No lamps

\section{Instrument \\ Operation}

Continues

operation

Instrument

disabled

Continues

operation

Instrument

disabled

Instrument

not energized

Continues

operation

Instrument is

in standby

Continues

operation
Operator

Intervention

Desired

Is a must

Should be

necessary

Is a must

Check cause of power off

Requested (Check pulse lines)

Is a must

Not immediately necessary

B. C. stands for building computer. 


\section{BLOCK DIAGRAMS AND ANALOG CHAINS}

The Effluent and Sanitary Sewer Monitor block diagrams are shown in Figs. 2 and 3 , respectively. Both analog chains $f$ i $i$ the Effluent Monitor (Fig. 2) contain the same the of units. The two detectors are the $\mathrm{Nal}$ type and have different dimensions to match the different ranges of energies described in Ref. 1. The units used in both chains are

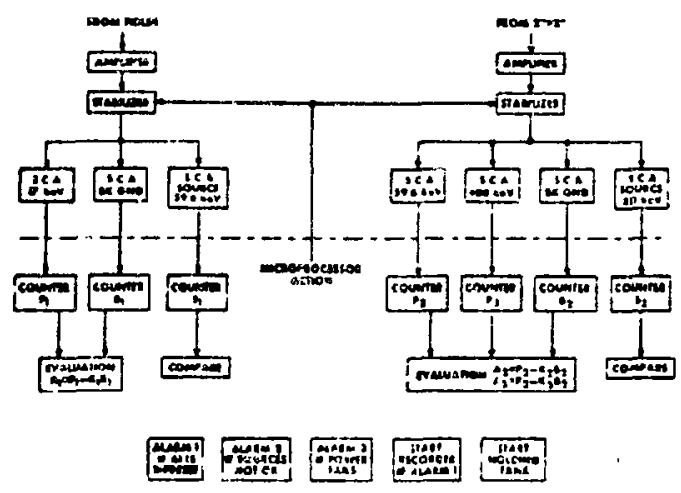

Fig. 2.

Block diagram of Effluent Monitor.
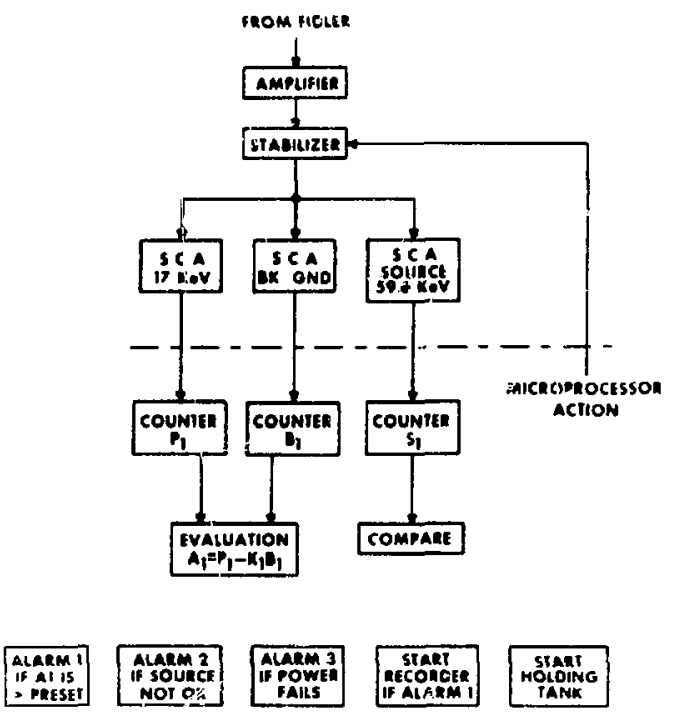

Fig. 3.

Block diagram of Sanitary Sewer Monitor.
- Preamplifier, "Model TC115A (Ternelec),

- Double Amplifier, Model 221Q (Kiksort).

- Single-Channel Analyzer (SCA), Model 550

(Ortec),

- Stabilizer and SCA, Model NA 22 (Harshaw), and

- High-Voltage Supply, Model AEC-315B (Power Degignj.

The units are mounted in a NIM bin and have coaxial cable connections for the analog signals. The two SCAs, indicated in Fig. 2 for the sources, are contained in the stabilizers. The seven outputs from SCAs are connected to the rear panel of the monitor by coaxial cables with LEMO-type connectors. The stabilizers have an additional coaxial connector that controls the operation from the CPU. This connector was added to the rear panel and is linked to test point TP1 18 of the NA-22 AGC Controller. The connection is parallel to the manual switch HOLD/TRACK, which must be set to the TRACK position. TRACK means open-circuit, so the gate control will pull up and enable the stabilizer operation. Normally, the Effluent Monitor pulls down this voltage, the gates are disabled, and the stabilizer is in HOLD condition. During the 1 -min STABILIZE time, the gates are enabled and the instrument can track and compensate for drifts. At the same time, the source counters receive pulses from the SCAs. After this time, the gain value achieved by the stabilizer is kept constant and the source counters are gated off until the next calibration.

The Sanitary Sewer Monitor has an identical structure except that only the FIDLER Nal detector is present. A single chain is used with two counters for $17 \mathrm{keV}$ and background plus a source counter using an ${ }^{201} \mathrm{Am}$ source for $59.6 \mathrm{keV}$, as for the first chain of the Effluent Monitor. The second source for the Effluent Monitor is a ${ }^{22} \mathrm{Na}$ source providing an energy radiation of $511 \mathrm{keV}$.

An analog nutput is provided by the instrument by a digital-to-analog converter (DAC). This analog signal is sent to a chart recorder that starts automatically if excessive radioactivity is diagnosed.

- The preamplifiers are mounted directly on top of the photomultiplier tubes and are not indicated in the block diagram. 


\section{HARDWARE GENERAL FEATURES}

The complete schematic is shown in Figs. 4, 5, 6, and 7 . The schematic is identical for both instruments even though many components are not necessary for the Sanitary Sewer Monitor and, in that case, it is redundant. The adoption of almost identical hardware for the two systems allows for perfect interchangeability and for storing a reaerve board for either system. In effect all hardware shown in Figs. 4 to 7 is contained in a single board, which has components assembled with high density and connections made using a three-level wire wrapping. The program for the wire-wrapping machine was developed in LASL Group E-4, using only two of the three available wire-wrap levels. The third one can be used profitably for tests or modifications.

The only hardware difference between the two systems is the use of two solenoids instead of a motor for driving the source in the second system. The differences in the connections are outside the board and are explained later (Figs. 8 and 9). The four following sections refer, respectively, to the four schematics shown in Figs. 4 to 7.

\section{MEMORY-MAPPED LOGIC}

Figure 4 shows the hardware related to memory mapped logic. Table I indicates the memory addresses assigned to the different parts of the memory. The on-board PRCIMs and RAMs have addresses already assigned by the manufacturer of the single-board computer. The other addresses were chosen to reduce hardware decoding to a minimum. So, bit 14 of the address bus ( 16 bus lines named $\phi$ to $1 \tilde{0}$ ) is used as a chip select for C-MOS RAM without complicated decodıng. Bit 15 is used to gate the addresses for source counters and operative fields. The first four addresses in the $8000 \mathrm{H}$ range (Table $\Pi$ ) are used for the source counters (Fig. 6). The operative fields have addresses 8004 through 8007 . Address decoding is performed by U2-1 (Fig. 4) using bit Al5 as a chip enable. The four addresses for operative fields are gated by U5-5 for "read" and "write" OP1 and OP2 (W8004, W8005, R8004, R8005) and by U1-11 (A and B) for "write only" Op3 and OP4. The latter are used to write AL1 and AL22 as 1 or 0 at the end of each measurement or at the end of the calibration, respectively. The written number will remain until the end of the next corresponding phase.

Operative field 1 (OP1) contains information about the phase to be executed and has address 8004H. Read (R8004) and write (W8004) de not always coincide. For example, bit $\phi$ canrot be writ ten but is only read from the switch APR, and it is used at the power-on as a decisional feature to go to halt (if $\phi$ ) or to start the operation automatically (if 1).

The data bus is memorized in U2-4 during the "write" operation. (Negative logic is used for both data and address buses.) The status of U2-4 plus switch APR and Alarms 21 and 23 can be read, sending information to the data bus via the tristate buffer U2-3 and U2-2B. A very particular operation is obtained by writing bits 3 and 5 . Their effect depends on the status of flip-flops MOV1 (U3-1B) and MOV2 (U3-2A). These flip-flops are set by W8004 ANDed with bits 2 and 4, respectively, in gates U3$3 \mathrm{~A}$ and U5-3C. They start surce movement by energizing the motor (Fig. 8). When the motor reaches its correct position, the flip-flop (MOV1 or MOV2, respectively) is reset. The next phase will send bits 3 or 5 , respectively. Bit 3 is routed through gates U.3-6A and U3-7A to set the source count flag (SCF) or to set ALARM 21 depending on confirmation of the movement on flip-flop U3-1B. Bit 5 is routed through gates $\mathrm{U} 3-6 \mathrm{D}$ and $\mathrm{U} 3-7 \mathrm{~B}$ to set tice measurement flag (MEASF) or to set ALARM 23, depending on confirmation of MOV2 on flip-flop U3-2A. WARMUP, MOV1, and MOV2 correspond directly in writing and reading.

All hardware operation is controlled by the ENABLE flip-flop (U3-9A). This is set by writing bit 6 at the address $8004 \mathrm{H}$ (OP1) and reset by writing bit 3 at the address $8005 \mathrm{H}$ (OP2). The key flip-flops and counters are reset when $E=0$ (ENABLE flip-flop reset).

ENABLE flip-flop is set when the operator gives the command START or CONTINUE. These commands write the values $44 \mathrm{H}$ and $60 \mathrm{H}$ in OP1, respectively, (Table $\mathrm{I}$ ) and set MOV1 or MEAS phase together with $\mathrm{E}=1$. Bit 6 , corresponding to set $\mathrm{E}$, is not read. This bit is used to read AL21, as expiained, whereas bit 7 is used only to read AL23.

The bits of OP2 are used as follows. Bits 0 and 2 set or reset TEST flip-flop (U1-8B) and PRINT flipflop (U1-2A), respectively. These flip-flops are always reset by the program at the beginning of each 


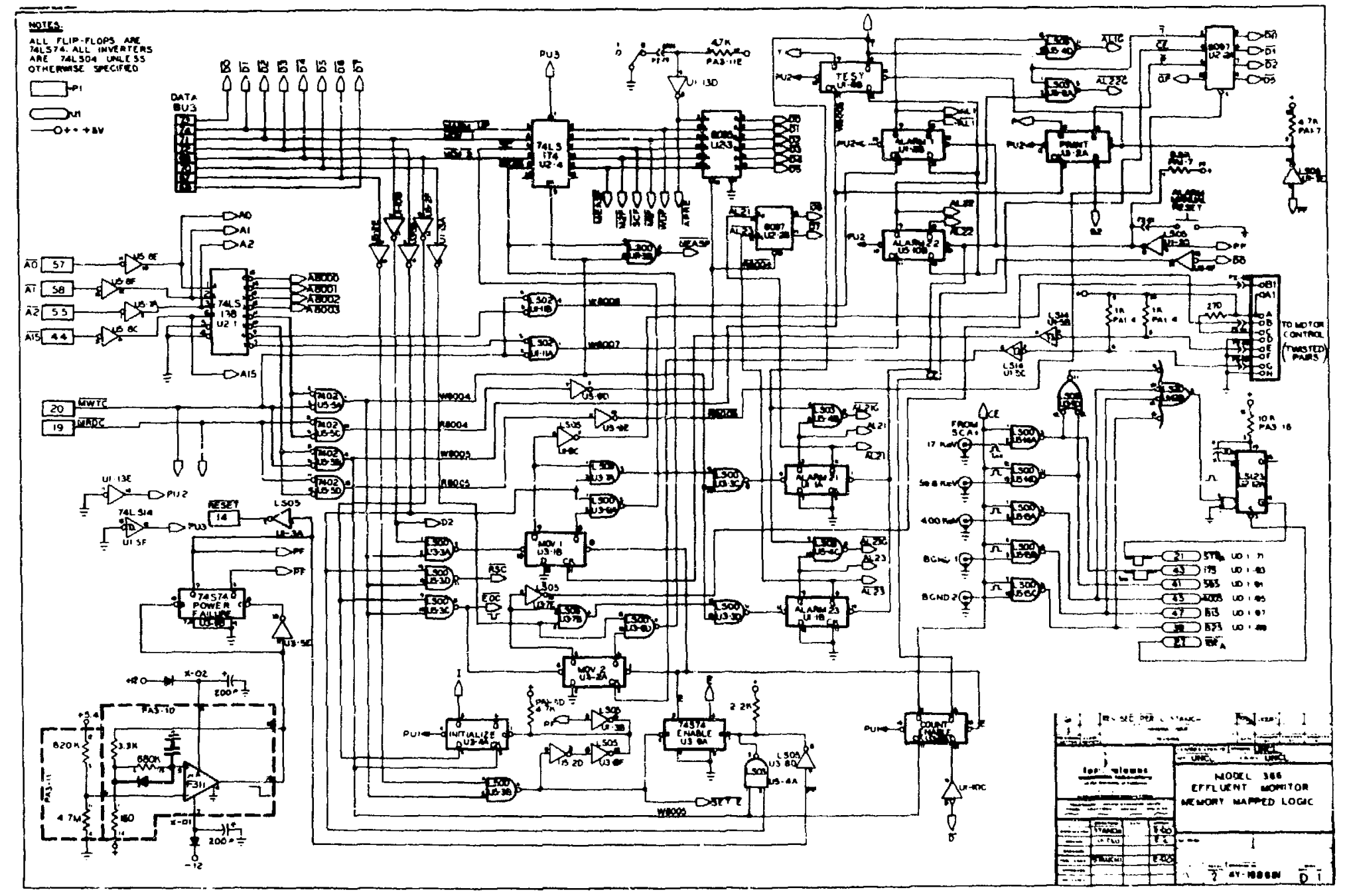

Fig. 4.

Detailed schematic, sheet 1 . 


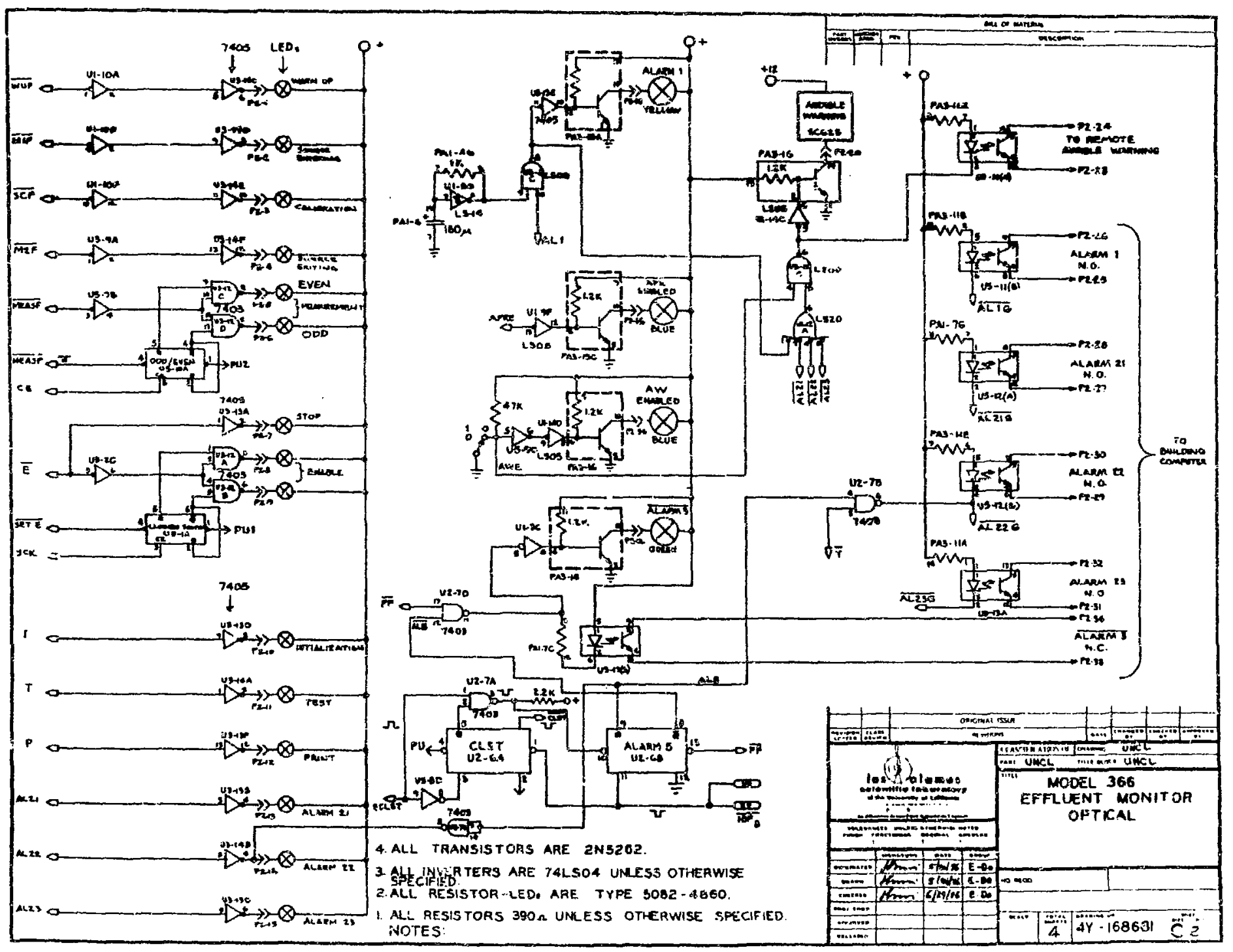

Fig. 5.

Detailed schematic, sheet 2 . 


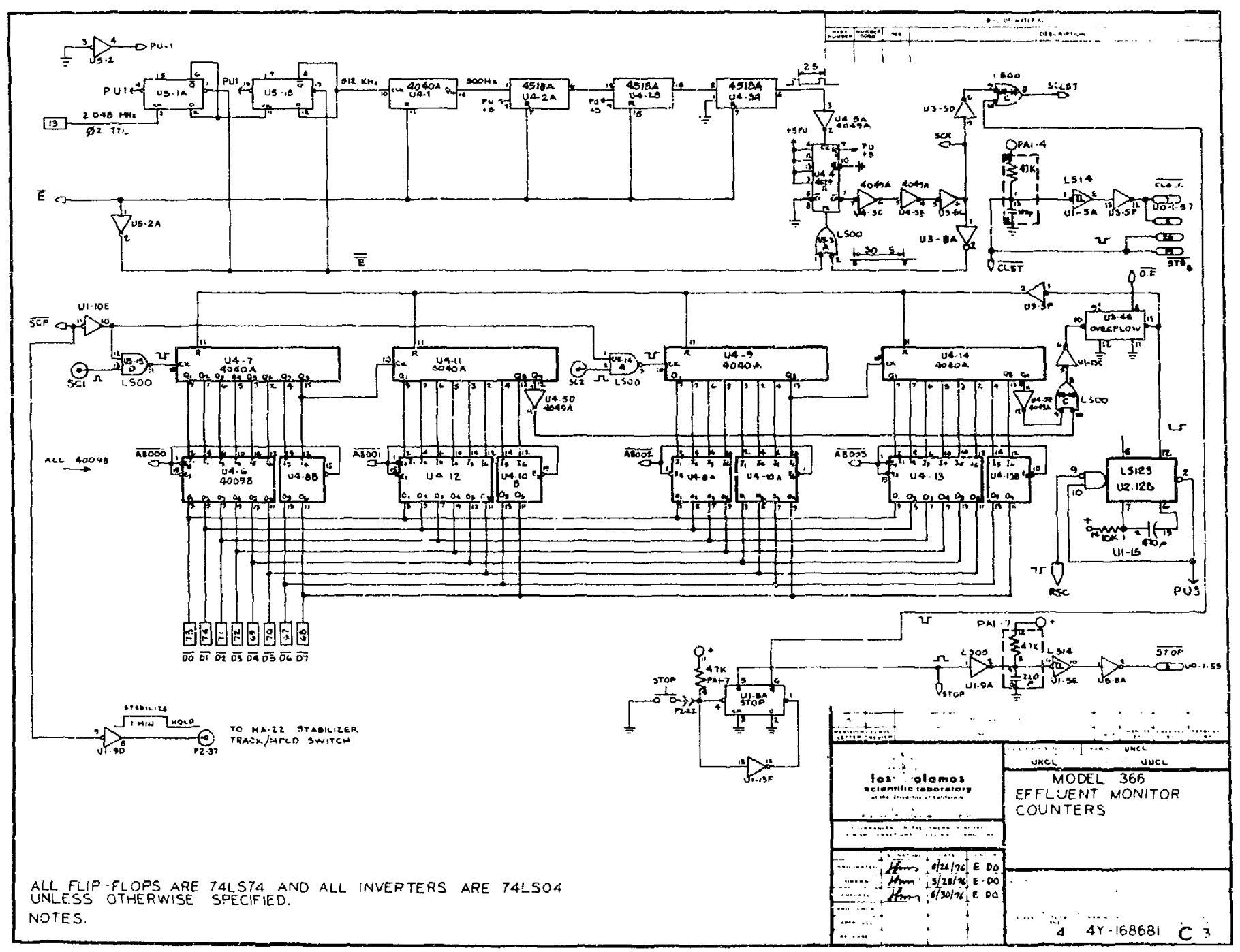

Fig. 6. 


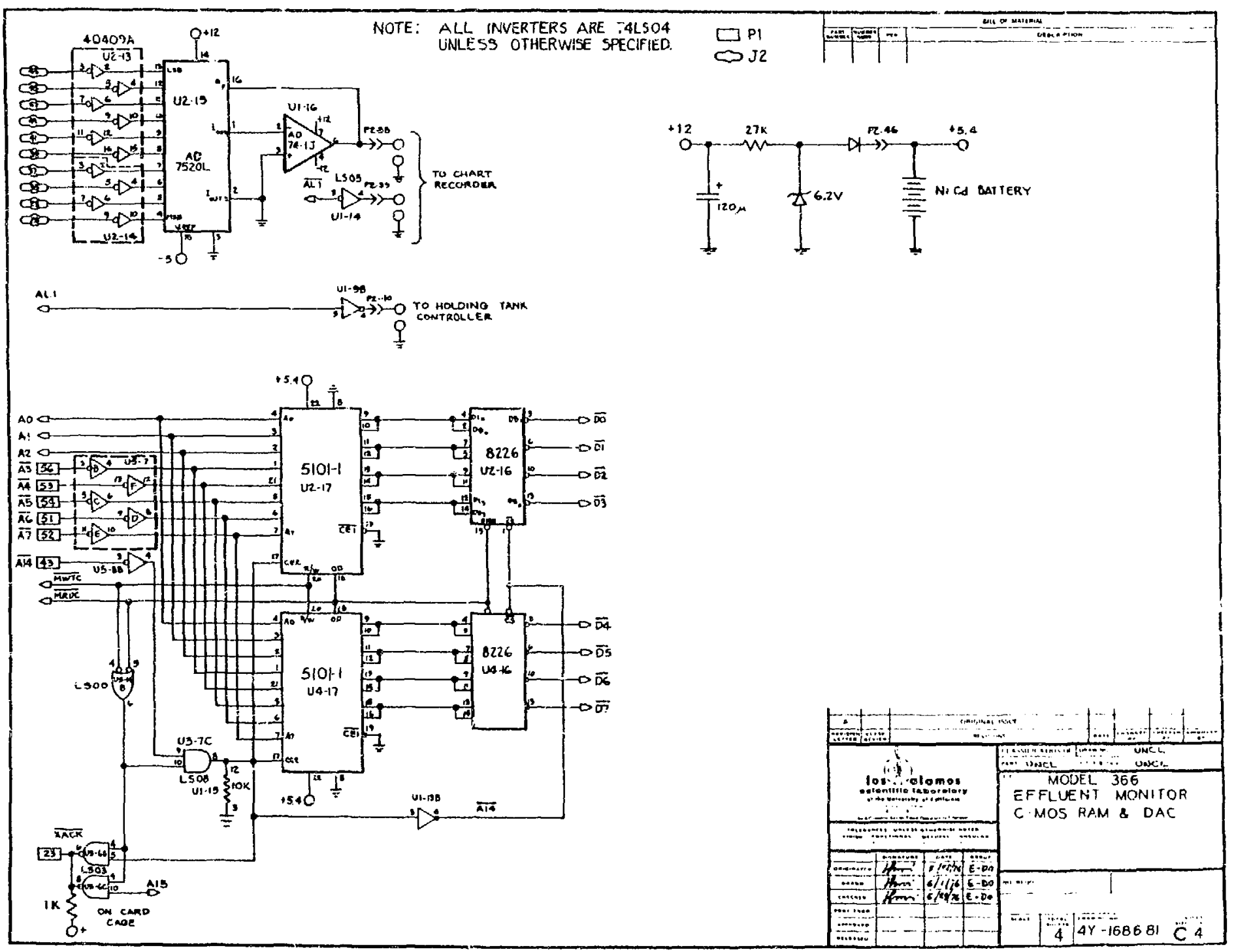

Fig. 7.

Detailed schematic, sheet 4 . 


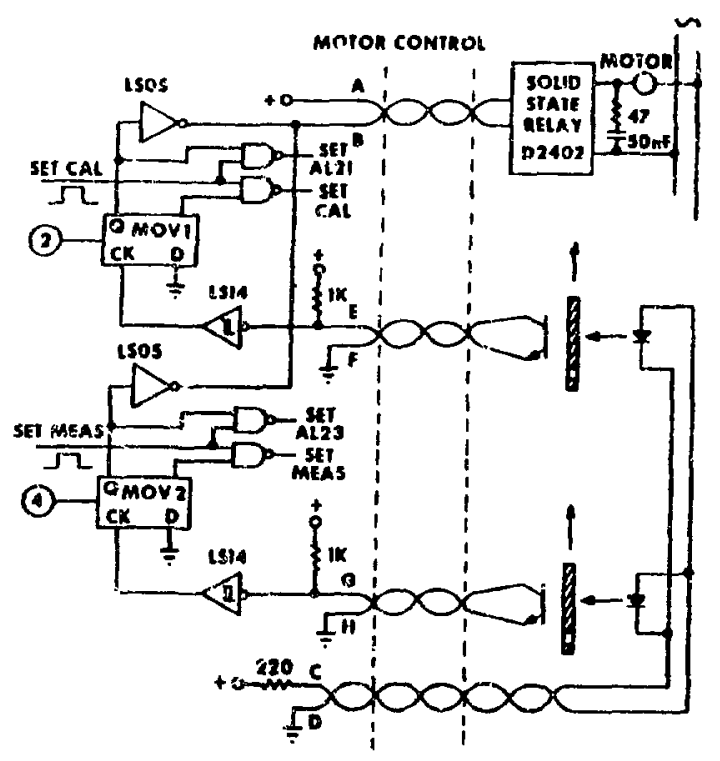

(2) stant MOVEMERT I (SOURCE EN1ELHO)

(4) stant movement 2 (sounce extmwo)

Fig. 8.

Motor control for the Effluent Monitor.

measurement, writing $\mathrm{OP} 2=\mathrm{O} 2 \mathrm{H}$. This value (bit 1 at address $8005 \mathrm{H}$ ) sets COUNT ENABLE (U3-2B) and resets TEST and PRINT. Bit 4 of OP2 is used when INITIALIZE is called by the operator to light the lamp indicating that phase. The program, when nperator types INITIALIZE and CR or simply I and CR, writes $O P 2=18 \mathrm{H}$, obtaining the dual action of disabling the hardware (atandby phase) and switching on the lamp INITIALIZE and the lamp STOP. ENABLE lamps are switched off. Bits 3 and 4 , corresponding to the value $18 \mathrm{H}$ in OP2, are not read. The only other bit that is read is bit 5 , corresponding to $20 \mathrm{H}$ (source count overflow, Fig. 6 ), which is sent to data bus together with $T, P$, and CE via the tristate buffer U2-2A.

Figure 4 also contains a power failure circuit based on the amplitude comparator $\mu$ AF311. This has a high-impedance FET input so that it can be connected to the $5.4-\mathrm{V}$ battery used for C-MOS RAM. The output of this comparator (pin 10) is normally high, and it becomes low in case of power failure. This happens if the +5 voltage drops below about $4.5 \mathrm{~V}$ and the output returns to high when the voltage is higher than $4.75 \mathrm{~V}$.

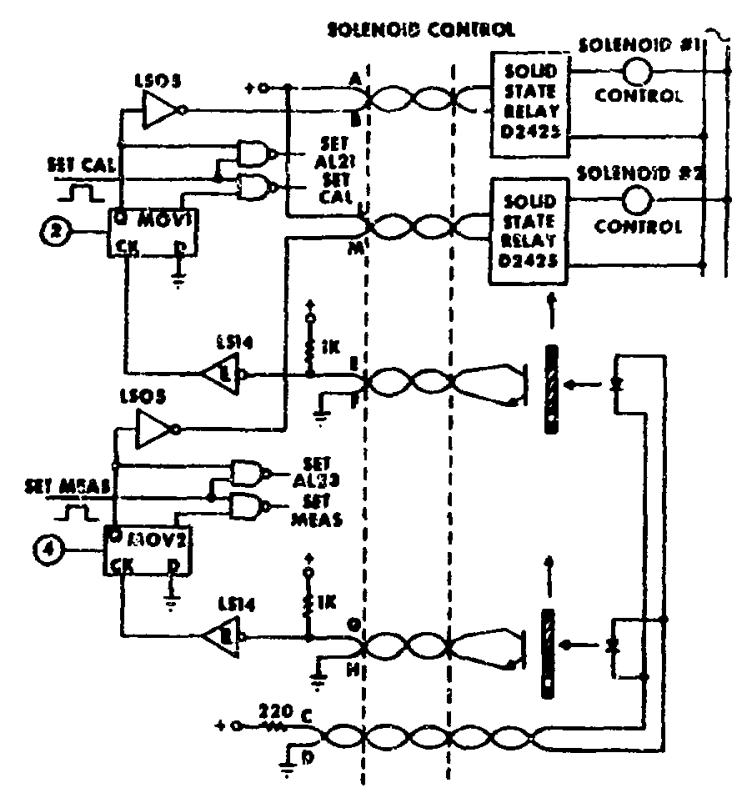

(2) SIARt movemtMr I (BOJHCR ENTEkmo) (4) sJant movemsht 2 (SOURe! Exmmo)

Fig. 9.

Solenoid Control for the Sanitary Sewer Monitor.

The noninverting input of the comparator has a 20-nF capacitor connected to ground to allow the \pm 12 voltages to build up before the +5 voltage is sensed during power-on. When power goes off, decrease of the +5 voltage is sensed rapidly because the $680-k \Omega$ resistor is short-circuited by a diode having the cathode connected to the $160-\Omega$ resistor.

The POWER FAILURE flip-flop U3-9B is tc set and reset by the output of the comparator. In normal operation its output $\mathrm{PF}$ is low and $\overline{\mathrm{PF}}$ is high. During power-up, PF increases with the supply voltage and is used to reset ENABLE and all alarm flip-flops as an initial condition. The same signal is used to reset the $C P U$ through contact 14 on the $P 1$ connector of the computer board.

The pulses coming from the five SCAs are gated by COUNT ENABLE flip-flop U3-2B. The Effluent Monitor has all five SCAs connected to inputs. The Sanitary Sewer Monitor has only $17 \mathrm{keV}$ and BG1 connected. The pulses are ORed by U3-7D and U112B. The OR drives the one-shot multivibrator $\mathrm{U} 2$ $12 \mathrm{~A}$, which is used like a flip-flop due to the long 
time constant. Its output sets the interrupt on the CPU, strobing the PPI with the high-to-low transition on $\mathrm{STB}_{\mathrm{A}}$. The one-shot multivibrator is reset by the handshaking action of the CPU via the negativegoing pulse IBF (Fanut Buffer Full). The CPU reads the data on the PPI and resets $I B F_{A}$.

Unused inputs cannot be left open because they will completely jam the instrument. As soon as COUNT ENABLE = 1, any open input (e.g., pin 13 on U15-14D) sets high the sutput of the OR U1-12B that drives the one-shot multivibrator U2-12A. This is reset by the acknowledgment $\mathrm{IBF}_{\mathrm{A}}$, but it is im. mediately restarted by the dc enabled input, causing a train of pulses during the total measurement time. This effect will not occur if negative logic is used for transmitting pulses, but we used the positive pulses directly as they were generated by the analog units. Connections to gates U5-14D, U5$15 \mathrm{~A}$, and U5-15C are shorted to ground at the cardcage connector in the Sanitary Sewer Monitor.

The difference between the two instruments concerning the source driving is explained by Figs. 8 and 9. The motor used for the Effluent Monitor ${ }^{1}$ has a unidirectional rotation that drives the sources from rest to operative position or vice versa with a $180^{\circ}$ rotation. For that purpose, both MOV1 and MOV2 drive the same winding of the motor, being ORed with open collector inverters 74LS05 (Fig. 8). The effective connection is not made on inverters U1-9C and U3-7E but is made outside the board on connector P2, shorting pins B and B1. in the block indicated as MOTOR CONTROL. This allows the two independent commands to be sent to the two solenoids of the Sanitary Sewer Monitor (Fig. 9).

The last feature shown in Fig. 4 is the ALARM MANUAL RESET used by the operator to reset all the alarms if necessary (especially during test).

\section{OPTICAL AND ALARM CONNECTIONS}

Figure 5 shows the connections to the front panel lamps, the galvanically isolated connections to the building computer, and the handshaking circuit for the clock.

The circuit for the lamps is quite evident. All the drivers are full-power open-collector inverters (type 7405) that drive the LED red lamps for phases and status indication. Two D-type flip-flops are used to switch the ENABLE lamps continuously at each clock and the MEAS lamps at each new measure14 ment. These four lamps are driven by four open collector gates (type 7403). The STOP lamp is driven by $\bar{E}$ via the inverter U3-12A so that it indicates that hardware is disabled $(\mathrm{E}=0)$. The high currents required by the ALARM 1, APRE, AWE, and $\overline{\text { ALARM } 3}$ lamps are supplied by buffer transitors. The ALARM 1 lamp is driven by a squarewave oscillator obtained with a feedback connection on the Schmitt inverter U1-5D. The result is that the yellow lamp flashes and is thereby more impressive than the other lamps. The alarms are ORed together and drive an audible warning device mounted on the front panel. A whistle is heard in case of any alarm, and the sound is intermittent for Alarm 1. The frequency of the square wave for Alarm 1 is about $10 \mathrm{~Hz}$. The audible warning is multiplied remotely via an optical isolator. If Alarm 1 is encountered with any alarm of group 2, the sound is continuous.

The circuit for the CLOCK and STOP handshaking uses a double flip-flop U2-6. The SCLST pulse (from Fig. 6) sets flip-flop CLST (U2-6A) with its trailing edge. The same pulse is presented to gate U2-7A, which is blocked by flip-flop CLST. When the flip-flop is set, the gate is enabled, but the pulse is already gone. The $\overline{\mathbf{Q}}$ of CLST flip-flop is sent as $\overline{\mathrm{STB}}_{\mathrm{B}}$ to the PPI, which, with a small delay, sends back its acknowledgment IBF $_{\mathrm{B}}$, resetting the flipflop. If this acknowledgment is not received, the next clock pulse will find the gate open and will set ALARM 5 flip-flop. This in turn simultmeously sets Alarms 22 and 3, sending the double information to the building computer and turning ALARM 3 lamp off and the ALARM 22 lamp on. An open-collector gate (U2-7) has been used to drive the larnp and remote driver for Alarm 22 as wired-OR. $\overline{A L 5}$ drives an AND connection, with $\overline{\mathrm{PF}}$ the Alarm 3 indication. Normally, this operates, but the absence of either input eliminates the drive so that Alarm 3 is sent to the building computer (N.C. connection), and the green lamp is switched off.

The double flip-flop operation for ALARM 5 is provided to avoid any timing problem between the duration of SCLST pulse and the arrival of $\mathrm{IBF}_{\mathrm{B}}$. $\mathrm{IBF}_{\mathrm{B}}$ should arrive before the end of the SCLST pulse so that normally the double flip-flop is redundent.

The other links to the building compucer are N.O. connections and are driven by the proper alarms gated by the TEST flip-flop. 


\section{CLOCK CIRCUITS AND SOURCE COUNTERS}

Figure 6 shows the clock circuit plus the source rounters with their tristate buffers. A quartzgenerated clock is taken from the SBC $80 / 10$ board and divided via a C-MOS chain down to the $0.5-\mathrm{Hz}$ frequency at the output of U4-3A. The input frequency is not accopted by the C-MOS dividers operated at $5 \mathrm{~V}$. Typically, they operate at a slightly higher frequency, but the minimum guaranteed is somewhat lower. Two D-type flip-flops divide this frequency to an easy value of $512 \mathrm{kHz}$. The 2-8 pulses from U4-3A are sent to the presettable divider U4-4 connected for binary operation and down counting. When the PE (Preset Enable) is set, the number 15 is entered from the parallel input. Af. ter 15 pulses, the content is zero and the $\overline{\mathrm{CO}}$ output goes low. A suitable loop returns the pulse to the $\mathrm{PE}$ input so that the content $\mathbf{1 5}$ is reestablished. One of the 16 states is skipped. The circuit divides by 15 , providing the clock of the instrument, that is, $30 \mathrm{~s}$. A stretching circuit provided by an RC of about $1 \mu \mathrm{s}$ and the Schmitt inverter U1-5A assure that the clock is longer than the strobe. ${ }^{*}$ Strobe $\mathrm{STB}_{\mathrm{B}}$ and the clock are sent to the CPU via a double contact on opposite sides of the connector.

The source counters are two 16-bit C-MOS coun. ters made from $4040 \mathrm{As}$. Counters UA .7 and U4-11 in series provide the necessary 16 bits for the $\mathrm{SC1}$ counter. A 17th bit on output Q9 of U4-11 is used to set the OVERFLOW tlip-flop. The same is true for counter SC2, using output Q9 from U4-14.

The outputs from the counters are sent directly to the data bus via the C.MOS tristate buffers (type 40098 ), which can be driven directly by the C-MOS counters and which have sufficient power to drive the data bus.

The source counters are enabled by the control flag SCF, which also drives the TRACK/HOLD switch on the Harshaw Na22 stabilizer. The reset of the counters is sent by the CPU as RSC via a oneshot multivibrator that provides a longer pulse. (The pulse time from the CPU is insufficient to reset the cascaded counters.)

Figure 6 also shows the STOP command, which acts through the interrupt, not directly on the

-The time constant has been abtained with $4.7 \mathrm{k \Omega} \times 220 \mathrm{pF}$. Note that the value of $100 \mathrm{pF}$ in Fig. 6 has been replaced with $220 \mathrm{pF}$. Drawing $4 Y-168681 \mathrm{hag}$, in elfect, this value. hardware, and sends the strobe $\mathrm{STB}_{\mathrm{B}}$ in $\mathrm{OR}$ with the clock.

\section{C-MOS RAM AND DIGITAL-TO-ANALOG CONVERTER (DAC)}

Figure 7 shows the C-MOS RAM circuitry plus the digital-to-analog converter. As explained before, C-MOS RAM is used to store the operative constants in low-power memories. These memories can be supplied for a long time by battery if the power fails.

The unique bit Aly flom the address bus is used as a chip select for C-MOS RAM. The chip select CE1 is grounded, and CE2 would be sufficiently gated by A14 if the single-board computer had a different design. However, a gating with the OR of "memory read" and "memory write" pulses (MRDC $+\mathrm{MWTC}$ ) is compulsory. Because the single-board computer energizes all 16 address lines when the CPU is in HALT, C-MOS RAM draws a large amount of dc current most of the time if it is not gated. The single-board computer design relies on the high-impedance state of the address bus from the CPU only for internal use. The external address bus is driven by buffers (type 8226) that drive all address lines when the $C_{+}^{P U}$ is disabled (highimpedance state). To avoid this inconvenience, the single-board computer controls the enables of the 8226 buffers in HOLD condition so that the addresses are at high impedance. Unfortunately, the Effluent and Sewer Monitors do not use HOLD, and the single-board computer does not gate the buffers with HALT.

The 8 least significant bits of the address bus are connected to C-MOS RAM, which can store 256 bytes. In practice only the first 16 bytes are used.

Figure 7 also shows the circuit for driving the chart-recorder analog input in case of alarm. The DAC-type AD 7520L receives the eight bits of the second PPI, port A (or port 4 in the SEC 80/10), and the two least significant bits of port $C$ ior port 6 in the SBC 80/10) that are connected as the two most significant bits (from connector $\mathrm{J} 2$ pins 23,25 ). The output is connected to the chart recorder via the operational amplifier U1-16. Simultaneously the alarm condition AL1 drives the normally idle motor of the chart recorder. 
CLOCK reads operative field OP1 calling the appropriate phase. On finding bit $1=1$, CLOCF calls WARMUP. Then the different operative phases follow one after the other in a preestablished order. If the operator does not press the CONTINUE com. mand, the succession of phases called by CLOCK looking at the value of OP1 is as follows: WARMUP, MOV1, STABILIZE, MOV2, MEAS,... MEAS, MOV1, STABILIZE, MOV2, MEAS. . MEAS, MOV1... The cycles continue endlessly. Each phase sets at its end the value of OP1 for the next phase, therefore, the succession is automatic. The operator acts on this succession by presetting the number of consecutive MEASs and their duration. He can always interrupt the cycle by pressing STOP or setting a command from the keyboard. (The latter is not accepted during the calibration sequence.)

A detailed explanation of the PL/M procedures, listed in the same order as the program, is reported below. Reference is made to EFFLU, but no conceptual difference is encountered in SEWEFF.

PC, PS, PSCR, and PN are used to give output to the teletype via the USART contained in the singleboard computer. They all refer to PC (Print Character), which has a "do while" that waits for USART to be ready and checks its status port continuously. When this port (Hex address ED) is 1 , the character is sent to the output port (Hex eddress EC). PS and PSCR print a previously declared message. PSCR adds to the message a CR, LF (Carriage Return, Line Feed) and a sufficient number of rub-out codes (7FH) to give the teletype time to execute $\mathrm{CR}, \mathrm{LF}$ (Message 13). These rub-out codes are especially necessary if a faster printer is used. $P N$ is somewhat more complicated; it is used to call a number stored as a hexadecimal number of two or four digits and to print it as a derimal number. At the end of the procedure, Message 19 types a dash (-) and two spaces that are particularly useful during initializa. tion.

IC and INS are used as input procedures from the USART. IC (Input Character) waits for USART to be ready and checks for value 2 of the status port. When USART is ready, the input is performed and immediately echoed to output so that the operator can see what he types. INS (Input Number String) is the reverse of $\mathrm{PN}$ and is used to acquire decimal numbers and to store them as hexadecimal num. bers.
INITIALIZE, described in Sec. II, acquires the operative constants and stores them in C-MOS RAM. Each constant is requested by the proper printing of a message. INS can set $\mathrm{STO}=1$ when a number is typed or STO $=\phi$ when $\mathrm{X}$ is typed. (In effect the procedure responds in the same way if only $\mathrm{CR}$, instead of $\mathrm{X}, \mathrm{CR}$, is typed.) INITI ALiZE stores the new number if it finds $S^{\prime}-U=1$; if not, it proceeds to the raxt message. The first message requests the measurement time STOMELE (Stored Measurement Length). When the first message is called, the stored number is divided by 2 before it is printed, and when a new number is typed by the operator, it is multiplied by 2 before it is stored. This allows the operator to type the time in minutes instead of in number of clock cycles. The other messages are as follows. STONUM means the number of measurements stored in a run before going to a new calibration. STOTHRESH fixes the maximum number of pulses accepted before ALARM 1 is set. STOL1, STOH1, STOL2, and STOH2 set limits for source counting. STOK1, STOK2, and STOK 3 are values of the constants $K$ used to evaluate the peak areas, multiplied by 100 . STOPRINT sets the number of successive measurements to be printed when the operator types PRINT. This constant is in normal RAM, and in case of power-off, the value is lost and set to 1 by POWERON. STOCORR is the additive constant for evaluation with Eq. (2).

START, CONTINIE, TEST, PRINT, STOP, EXPER, and OPER were described in Sec. II.

COUNTSTART is used to set the proper conditions for starting a measurement. The five counters are reset, the measurement time is set equal to STOMELE, and the overflow indicators are reset. Operative field OP2 is set to the value of 2 , which meanis set COUNT ENABLE and reset PRINT and TEST flags as explained. This procedure is called whenever a measurement is started, that is, by CONTINUE, by end of MOV2 after calibration, and by end of MEAS tc continue the series of measurements in one run. There is a small difference in the calling. The first two calls add NM=STONUM. that is, they fix the number of measurements to be done equal to the stored number STONUM. When COUNTSTART is called by the end of measurement, the value of NM is not fixed to the initial 
value, but it is left at the number reached by decrementing it at each measurement.

IB (input byte) is used to acquire the bytes in machine code for procedure OPER (see Ref. 2).

PULSES is called when a pulse is received by the input PPI from one of the five SCAs connected to it via the gates enabied by COUNT ENABLE during the MEAS phase. When a pulse is received, it is stored by incrementing the corresponding counter. If one of three peak counters arrives at the maximum content, the overflow indicator (OVERSEV or OVERFIF or OVERFOH, respectively) is set to avoid other pulses overflowing the counter and eventually displaying a small number.

WARMUP is called by the first clocks after POWERON if APRE $=1$. The warm-up time WT is decreased by each clock and $W$ is decreased when $W T=0$. Number $W$ is set $=2$ by POWERON and allows two successive runs of WARMUP if the first calibration fails. WnE.J $N T=0$ WARMUP sets OP1 $1=4$, that is, sturts MOV1.

MOV1 should find the insertion of the sources in the calibrating position. Some variables like NOWPRINT. PT, and PRINTEXEC (but not PRINT flag) used to print data from counters are reset at the beginning of the MOV1 procedure. (Note that MOV1 does not start the movement but should terminate the 30 a allowed for this phase.) If the moverient has already been confirmed by hardware, MOV1, after setting $\mathrm{C} 1=2$, that is, $1 \mathrm{~min}$ for calibration, sets $O P 1=8$, which is the value for stabilize. If confirmation of the movement is lacking, the circuit sets AL21 as described in Sec. VII. This is read as OP1 $=40 \mathrm{H}$ and causes the output $\mathrm{OP} 2=8$ that means disable hardware.

STABILIZE starts the return movement when the second clock is received. OP2 is read at the end of the $1 \mathrm{~min}$ allowed for source counting to check if print has been requested by the operator or if overflow in either source counter occurred. Then the evaluation of counts is made, comparing each counter content with its prefixed low and high limits. If at least one of the limits is not respected or in case of overflow and if $W=0$, the procedure sets ALARM 22. If $W=1$, a new warm-up cycle is allowed. This occurs only at the power-on because during the endless succession of a run of measurements followed by a calibration the value of $W$ is always $O$. When first powered, one warm-up time of $10 \mathrm{~min}$ or two warm-up times for a total of $20 \mathrm{~min}$ are performed.
If the calibration fails at the end of the second warm-up, AL22 is set as during the normal succession of cycles and the flag for printing (PRINTSOURCE) is raised automatically. If the calibration is all right, the two statements $W=0$ and $A L 22=0$ are written at the end of the procedure.

MOV2 behaves like MOV1. MEAS flag is set $(\mathrm{OPl}=2 \mathrm{OH})$, and $\mathrm{AL} 23$ is read as $\mathrm{OPl}=80 \mathrm{H}$. If $A L 23=1$, then hardware is disabled, else the value of $W$ is checked to decide what phase should be next. If $W=0$, the next phase is MEAS, and this is intialized calling COUNTSTART and setting the proper number of neusurements (NM). If $W=1$, a new warm-up is ttarted by setting $O P 1=2$.

MEAS resets the print indicetur NOWPRIN' to avoid printing at each clock; then the measurement time (MT) is decremented. When $\mathrm{MT}=0$, one measurement is completed and COUNT ENABLE flip-flop is reset, blocking the incoming pulses. An indicator of background greater than peak (BIGBACK) is reset and will be set later if the corrected background $\mathrm{BG} 1{ }^{*} \mathrm{~K} 1$ is greater than the content of SEV counter. Protections against wrong evaluations due to overflows or to lack of pulses are performed by this procedure. If any line is cut off, the corresponding counter receives zero pulses. If there is no hardware inconvenience, each line should have at least some random pulses even with very clean effluent. A zero in any counter is considered a reason for Alarm 4, obtained by coding group 2 alarms (ALARM $4=$ AL21 + AL23). Printing is autcmatically performed setting PRINTEXEC $=1$. If any overflow indicator was set by PULSE, the corresponding area is set to FFFFH(65535). If none was set, the area is calculated with Eq. (1). The area corresponding to $17 \mathrm{keV}$ is calculated with Eq. (2). If the counter SEV, FIF, or FOH has zero content, its corresponding area is not evaluated and it is set to zero. If ALARM 4 is found and no zero is printed, this means that the alarm was set by a background content $=\phi$. If background is greater than the contents of the $17-\mathrm{keV}$ counter (SEV), then BIGBACK is set to 1 to print the suitable message. The constant STOCORR may prevent a negative result, but if it is insufficient, ALARM 1 is probably set by the large result corresponding to a negative number. The overflows themselves are no reason for alarm, but OVERSEV $=1$ sets Alarm 1 because area $A 17$ is set to FFFFH, which is undoubtedly greater than 
the preset threshold. PRINTEXEC is set in case of AL1 and in case of PRINT $=1$ (set by operator). When one measurement is terminated, the NM is decremented and NOWPRINT is set. A printout will be performed if the AND of PRINTEXEC * NOWPRINT=1. Then the next measurement is initialized calling COUNTSTART, or the next calibration is flagged, writing $O P 1=4$ when $N M=0$.

PRINTOUT is called by CLST when PRINTEX. EC * NOWPRINT $=1$. Print indicator PRINTEXEC is reset when $P T=0$. NOWPRINT is always set to zero when MEAS is called, and it is set to 1 only when a messurement is finished. This avoids printouts at the end of each clock during the measurements. Number PT is set $=1$ by ALARM 1 or =STOPRINT if operator types PRINT. Each call of PRINTOUT decreases PT so that a prefixed number of printouts is performed. Only one printout is available in case of alarm. The area of $17 \mathrm{keV}$ is divided by four as explained in Sec. $X$. This area, called PINTO, is set to FFFFH if the result of the division is greater than or equal to 1024 so the full scale is sent to the DAC. This avoids the possibility of having a small number in the least significant 10 bits plus some higher bit that would not be received by the DAC. The printout on the teletypewriter has a series of messages. First, message 18 (ME18) starts the motor of the teletype, sending a series of RUBOUTs. Then, if BIGBACK $=1$, the message BACKGND PEAK is printed. Finally, the areas are printed with the header PEAK AREAS:. The contents of the three peak areas are printed on one line.

SPROUT is called by CLST and behaves similarly to PRINTOUT, printing the source counters content when PRINTSOURCE $=1$. Only one printout is made, therefore number indicators like PT in PRINTOUT are not used here. The operation is like that above, started by ME18 (series of RUBOUTs) and by a header: SOURCE COUNTS:. The contents of the two sources are printed in two lines for the Effluent Monitor. PRINTSOURCE is set to 1 if the calibration is out of the predetermined limits or if the operator types PRINT. Note that this can be done during MOV1 or STABILIZE or during the lest measurement with the consequence of printing both the last measurement before calibration and the result of calibration.

CLOCK is called by CLS $\Gamma$, which in turn is called by TOTALBREAK at each clock (or in case of stop). The series of IFs in the procedure is reversed as to the succession of operations. This simplifies the procedure, it being necessary to write only one DO END group containing the return instruction. This deserves more explanation. CLOCK calls one procedure corresponding to a phase defined by OP1. The content of OP1 is set by the end of the called procedure and is found by the next clock. So each procedure sets the conditions for the next one, and the procedures follow each other like a daisy chain. The and of WARMUP sets OP1 for MOV1. This writes $O P 1=4$, which is used for STABILIZE. The end of STABILIZE sets OP1 $=1 \mathrm{OH}$, w!lich is used to cell MOV2. Finally, MOV2 sets $\mathrm{OP}=2 \mathrm{OH}$, which is used by clock to call MEAS. The program executes the series of IF statements in the order they are presented so that when a procedure is called (such as STABILIZE), it returns to the statement following the call point bringing the new value of OP1 $(O P 1=1 O H)$, corresponding to a procedure that is called by an above statement (CALL MOV2). So a double call is impossible. The only case where a double call is feasible is in the last MEAS of a series. In this case MEAS returns with OP1 $=04$, and CLOCK calls MOV1 without waiting $30 \mathrm{~s}$. To ave id this occurence the DO - END group containing the CALL MEAS returns directly to EXIT by the RET statement.

CLST is cailed by an interrupt and calls CLOC $\mathrm{K}$ or STOP. Bit $\phi$ of port B of the PPI (port address E5) indicates CLOCK and bit 1 indicates STOP. CLOCK is called both in the effective presence of bit $\phi$ and if no bits are set in port $B$. This case is considered as ALARM 6, a minor emergency, and should be recognized by the operator. It is assumed that if CLST is called by the interrupt and the operator does not operate tise STOP pushbutton, the only possible cause of the call is a clock. The program in this case behaves as if a clock bit was found and in addition $F$ ints a message "NO CLOCK." This message also can arrive in periods of idle teletype when it is preceded by ME18 (series of RUBOUT). The end of CLST returns to TOTALBREAK and then to EXIT after a check of the conditions for printing is made. If the AND of PRINTOUT with NOWPRINT equals 1, PRIN. TOUT is called, as explained before. If PRINTSOURCE $=1$, SPROUT is called. STOP is called if, and only if, bit $\mathbf{l}$ is set. Due to hardware in. version, this means port $B=F D H$. 
RECO is used to call procedures by recognizing the first letter of a procedure name typed by the operator. It is illegal to use the keyboard during calibration except for typing PRINT. The operator should wait for completion of this three-phase, 2min operation. To avoid interrupts when sources are in calibrating position or midway, if the operator types a command different from PRINT during the 2 min corresponding to MOV1 + STABILIZE + MOV2, the command is not executed and ILI EGAL COMMAND is printed. The OR of the three-phase operation is from Table II: 00011100 , that is, $\mathbf{1 C H}$. All commands are accipted at any other moment. If the first character is not one of the capital letters, A to $\mathrm{Z}$, a message 23 (ILLEGAL CHARACTER) is printed and no action is taken. If the initial does not correspond to one of the stated procedures, no action is taken and a message 15 (WRONG INITLAL) is printed. The legal case of a correct initial calls the desired procedure.

TOTALBREAK corresponds to the only level of interrupt allowed by the single-board computer. An input from PPI, port $\mathrm{C}$ (address $\mathrm{E} 6$ in the singleboard computer) is performed to recognize if a call was due to the PPI. First, a CLST bit is checked and, second, a PULSES bit is verified. If both are absent the interrupt was certainly due to the keyboard via USART. The RECO is called. The end of TOTALBREAK returns to the calling point, that is, EXIT. In effect, the normal state of the instrument is at EXIT, that is, HALT and the return from interrupt is to the location following HALT where a jump back to EXIT is found. TOTALBKEAK disables the interrupts so that the service of other interrupts when the phase is flipping from TOTALBREAK to CLST to CLOCK to one of the phases (MOV1 to MEAS) as a daisy chain is not accomplished. The return chain must be completed before the interrupts can be re-enabled by the return from TOTALBREAK to EXIT.

\section{USE OF SOFTWARE SIMULATOR}

The INTERPB simulator stored in the computing facility provides a first means to check a program associated with 8080 microprocessor. The simulator allows the designer to run the program step by step and trace the status of the CPU and the registers whenever desired. The simulator must be rold about the input/output ports and instructed about the reference points where the designer wishes the program to be stopped automatically. There are provisions for enabling and disabiing interiupts and simulating external occurrences so that the operator can set up an interrupt whenever he wishes. The designer can ask at any time that the program be executed to the next reference point or advance a stated number of steps. Display or alteration of memory locations is possible and the effect of a modification can be seen immediately.

The designer can use this tool to ascertain if the program is doing effectively what was intended. Access to INTEF.PB is gained by calling program SIMEF.

Program SIMEFF:

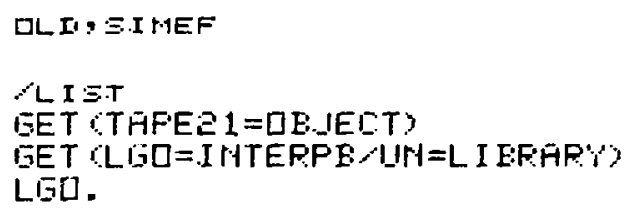

The total memory capability of the INTERPB is limited, and only $16 \mathrm{k}$ addresses are accepted (hexadecimal values from 0 to 3FFF). EFFLU and SEWEFF have higher addresses for C-MOS RAM and OP fields (see Table I). These addresses in the range of $4000 \mathrm{H}$ and $8000 \mathrm{H}$ must be replaced by editing the source program and calling the compiler for a new object program. The addresses used by the simulator are reported in Table IV.

The simulator proved to be very useful in verify. ing the different procedures and testing the proper succession of the phases governed by the value of operative fields.

The only drawback of this simulator resides in the fact that many instructions have to be given at the beginning. Also, the operation is quite long and tedious and it is slowed down considerably by the heavy time-sharing load of the computer. When work is stopped, all the initialization is lost so that many consecutive work hours are needed to exploit the initial efforts.

When all the program has been tested successfully, a necessary but still insufficient condition is achieved. The next step is to use the SBC 80P Monitor, which deals with the hardware and performs a more comprehensive test. 
TABLE IV

\section{ADDRESSES FOP UGS BY INTERPB SIMULATOR}

\begin{tabular}{ll}
$\begin{array}{l}\text { Hexadecimal } \\
\text { Addresses }\end{array}$ & Use \\
\cline { 1 - 1 } 0 & \\
$0000-$ OFFF & Program \\
$1000-100 \mathrm{~F}$ & C-MOS RAM \\
$2000-2007$ & Counters and OP Fields \\
3 C00-3CFF & RAM
\end{tabular}

Comment

As effective program

Addresses decreased

Mddresses decreased

As effective program

\section{USE DF THE INTEL MONITOR}

Intel provides a firmware monitor contained in two 1k-byte PROMS. This SBC 80P Monitor tests instruments based on the single-board computer SBC 80/10 and uses the first two sockets allowed for PROMs on the board.

The program to be tested, called "user program," must be modified to leave at least the first two addresses for the PROMs to the monitor, and it is stored in a RAM memory during the test period.

For the Effluent Monitor, a 16k dynamic RAM memory board (type SBC-016) is used because it can be inserted in the same cardcage that contains the single-board computer and the hardware board. The addresses of this board start at $0,4000 \mathrm{H}$, $8000 \mathrm{H}$, or $\mathrm{C} 000 \mathrm{H}$. Addresses between $1000 \mathrm{H}$ and 3BFF that are not used by either the monitor or the on-board RAM cannot be used with this $16 \mathrm{k}$ memory because they will jam the first addresses with the monitor. A 4k RAM memory can be used for this purpose, but we used the only available memory board. The addresses used are reported in Taible V. Note that the addresses for the variable memory are moved. Although the RAM memory assembled on the single-board computer has addresses $3 \mathrm{CO0}$ to 3FFF that do not interfere with any other port, the PL/M compiler does not accept addresses for the variable memory lower than those allocated to the program. So the RAM is also moved beyond the end of the program to the $5000 \mathrm{H}$ field.

The modified program is loaded into RAMs, using the monitor as a direct loader. The object code from the compiler is obtained on punched tape which is then read by a Teletype ASR 33 and loaded into the dynamic RAM.

The use of the monitor also poses other problems. The program has statements for USART initialization, but the monitor performs them before using USART. Thus, these statements are not recognized as Mode Format and Command Format when the user program gets control, so they must be removed. To maintain the strict correspondence with the final program and ensure the rigid displacement of each statement of $4000 \mathrm{H}$, some NOPs must be inserted, instructing the program in POWERON to disregard the USART commands and to start from a fixed ad. dress that takes care of the skipped instructions.

The declarations in the effective program start at address $3 B$, which is the first free address after the jump instruction contained at address $38 \mathrm{H}$ to serve Interrupt 7 . This address is assigned directly by the compiler, but when the monitor is used it must be forced with a numerical label to the value $403 \mathrm{~B}$. Moreover, some absolute GO TO statements must be replaced by adding $4000 \mathrm{H}$. An address to RAM must move from $3 \mathrm{CXX}$ to $50 \mathrm{XXH}$.

During tests it is convenient to have a system rezet independent of a power-off. A suitable circuit has been made, using a double-through pushbutton connected to a debounce circuit (two 7400 gates interconnected as a flip-flop) and a one-shot multivibrator generating a pulse of $100 \mu \mathrm{s}$. The circuit generates the required reset pulse, placing it in $\mathrm{OR}$ with the power failure detector via an open collector inverter connected to the output of the $\mu$ AF311 comparator (pin 10 of PA 3.10 in Fig. 4). Be cautious because it is easy to damage the following circuits if 
TABLE V

\section{ADDRESSES FOR USE OF SBC 80P MONITOR}

\begin{abstract}
Hexadecimal
Addresses

0000 - 07FF

4000 - 4FFF

5000 - 53FF

$6000 \cdot 600 \mathrm{~F}$

$8000-8007$
\end{abstract}

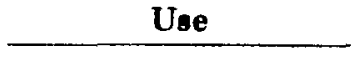

Monitor

Program into RAM

Variable memory

Simulated C.MOS RAM

Counters and OP fields

Comment
First -yo PROM sockets
Progi m displared by $4000 \mathrm{H}$
Replaces normal RAM
C.MOS addresses occupied
by program

As effective program the connection is made with the power on. The near pin 11 has a +12 voltage so it is preferable to make the connection with the power off. The reset circuit has been built on a breadboard, using a floating $5-\mathrm{V}$ power supply.

\section{A. Hardware Testing with 80P Monitor}

The Intel $80 \mathrm{P}$ monitor can be used to test the hardware independently of program control. The Substitute Memory Command (S) provides read/write acce $\leqslant s$ to desired memory locations, particularly operative fields. Insteed of giving here the exact order of testing, which depends on the problems encountered, some examples will illustrate the general method.

1. To test WUF (WARMUP flag):

Type: S8004 (SP) [(SP) indicates a space.]

The system responds: $\mathrm{XX}$ -

Now type: 2 (CR)

The WARMUP lamp should light.

Then type: S8004 (SP)

The system should respond:

02- (03- if APRE=1) indicating

that the warm-up flag is read.

2. To test the source counters:

S8004 XX- $\phi(\mathrm{SP}) \mathrm{XX}-8(\mathrm{CR})$

S8004 $\phi \phi-48(\mathrm{CR})$

The first statement disables hardware. The second statement sets $O P 1=48$, that is, sets
ENABLE flip-flop and enabies the source counters, resetting them in the process. The ENABLE lamps should come on (alternating every $30 \mathrm{~s}$ ) and the CALIBRATE lamp should light.

S8000 $\phi \phi-\phi \phi-\phi \phi-\phi \phi-08-(\mathrm{CR})$

The first four numbers indicate that the source counters have been reset. The fifth number corresponds to OP1, in which the previous command $48 \mathrm{H}$ has set the value 08 , that is, source count flag has been set. The last number (if not $20 \mathrm{H}$ ) indicates that the OVERFLOW flag has been reset.

Now place a number of pulses in source counter 1 . S8000 NN- NN- $\phi \phi-(C R)$

NN- NN- should equal the number of pulses placed in source counter 1 . Now, place a large number of pulses in source counter $2(>65535$ or FFFF $)$.

S8000 NN- NN- MM- MM- 0B- 20-(CR)

MM- MM- is the number of pulses entered in source counter 2 modulo 65536 . Number 20 at address 8005 (OP2) indicates overflow of the source counter.

\section{To test ALARM 21:}

S8004 XX-44(CR) (Start)

Before the movement of the source has been completed:

S8004 04-8 XX-8(CR)

The above statements set OP1 to 8 , that is, STABILIZE, before confirmation of movement and this sets AL21 and sets OP2 to 8, that is, disable as performed by the program in case of AL21. The 
ALARM 21 lamp should turn on, the SOURCE ENTER lamp turn off, and the STOP lamp turn on. Audible indication can be tested by turning on the AWE switch. A steady torie should be heard.

Other parts of the operative fields can be tested in similar feshior by using the $S$ command to write into the $O P$ fields and read the results.

The 80P monitor has no direct access to the $1 / 0$ port: of the SBC 80/10. These can be accessed by running a short program. To place a number in a port, place this sequence in memory:

$\begin{array}{ll}\text { OUT } & \text { DC } \\ \text { PORT } & \text { (PORT) }\end{array}$

RST1 CF.

Output is accomplished by setting the $A$ register to the desired number ( $\mathrm{XA}$ command) and then transferring control to the short program. Control will revert to the monitor at the end of execution.

Input from an $\mathrm{V} / \mathrm{O}$ port is similar. The program to be put in memory is

IN DB

\section{(PORT) (PORT) \\ RST1 CF.}

Input is accomplished by executing the program and then examining the contents of the $A$ register, using the XA command.

\section{B. Running User Program Stored in RAM Card}

Whenever major program changes are made, it is desirable to check these changes by running the program from RAM. To run with a RAM card, it is necessary to disable C-MOS RAM, which would occupy the same addresses on the interface board. To do this, remove the two 8226s (U2-16 and U4-16) from the interface board. Disable the RAM acknowledge by removing U5-6 from its socket, bending pin 6 out so that it does not make coniact and replacing it in its socket.

The RAM board can now be placed in the bottom slat of the cardcage and will function in place of $C$ MOS RAM. If the 80P monitor ROMs are placed in the $80 / 10$ CPU board, the program can now be run from the RAM card.

The Intel 80P monitor unconditionally enables interrupts before tranaferring control to the user program (EFFLU). Unfortunately, until PPI1 is in- * itialized, the lines used for interrupt requcst are left as inputs from the previrus reset. This leaves the lines fioating (high-impedance state) while being seen as a 1 by the following OR gate (A45, pins 5-6 on the $\mathrm{SBC} 80 / 10$ ). This creates an interrupt request and makes it difficult to transfer control to EFFLU.

Three methods can be used to over ome this difficulty. (1) The interrupt request line (pin 14 of the CPU / can be grounded through an external connection that is removed after PPIl has been initialized. More practically, the ground contact can be made on jumpers 46 and 49 connected to A45 pins 5-6. (2) The instruction C9 (RETURN) can be placed in location 3C3D, where control is transferred on interrupt (see Ref. 5, p. B-1), and a short preprogram written to replace this instruction with $\mathrm{C} 3$ (JUMP) before entering EFFLU (Table VI). (3) The enable interrupt instruction at address $339 \mathrm{H}$ in the monitor can be replaced by NOP ( $\phi \phi H)$, avoiding interrupt enable before transfer of control to the user program.

The second method based on the software patch (Table VI) deserves more explanation. The Intel 30) monitor has a JMP to address $3 \mathrm{C} 3 \mathrm{D}$ as a result of an external interrupt (INT7). The user program starts at address $403 \mathrm{~B}(3 \mathrm{~B}$, effective start of EFFLU beyond the space reserved for interrupts $+4000 \mathrm{H}$ ). The patch program arrives at one less than this adàress.

The execution sequence is

XS 3C $\phi \phi$ - 7FFF (Plece the stack pointer where there is more room.)

S3C3D C3- C9 (Place a return instruction instead of a jump into the address reeched with interrupt.)

G $4 \phi 36$ (Transfer control to the patch program.)

The routine for a GO command in the monitor is first to save the typed address on stack and second to make some operations on registers before starting the sequence $\mathrm{El}$, RET. The interrupt is immediately encountered as stated, so that a double return is consequently found, first in 3C3D and then in 33A, which is the location where the first return goes. The second return points to the typed address that was saved on stack and gives control to the user program's previous replacement of the 3C3D location with JMP to TOTALBREAK (C3, CC, 4F).

The third method has proved the mcst effective because most of EFFLU runs with the interrupts disabled. 


\section{TABLE VI}

\section{INTERRUPT SERVICE CONTROL FOR USE OF MONITOR SBC 80P}

Address Location
$3 \mathrm{C} 3 \mathrm{D}$


40336
40337
4083
40339
$403 \mathrm{~A}$
$403 \mathrm{~B}$

\begin{tabular}{l}
\multicolumn{1}{c}{ Initial Value } \\
\hline C9 Ret \\
(CC) No effect \\
(4F) \\
21 LXI H \\
3D \\
3C \\
36 MVI M \\
C3 \\
Start user program
\end{tabular}

\section{Final Value}

$\left.\begin{array}{l}C 3 \\ C C \\ 4 F\end{array}\right\} \begin{aligned} & \text { TMP } \\ & \end{aligned}$

\section{CONCLUSIONS}

The instrument, after tests of hardware and progran made with the sofiware simulator and the Monitor SP 80C, has been checked with its program loaded into $4 \mathrm{k}$ PROMs. An existing system for writing the PROMs was available, but unfortunately it was designed for smaller PKOMs. No means was available at the moment to load the Intel 8708 [ROMs directly, therefore we had to use an Exorciser Motorola that uses slightly different c.des. We hed availabie an Intel-Motorola translator plogram, an easy tool for obtaining the necessary object program. This program, OBJECTMOTO, was used directly with the Exorciser to burn in the PROMs anis the same has been done for the Sanitary Sewer Monitor with the translated program, SEWEMOTO.

Views of the Effluent and Sanitary Sewer Monitors are shown in Figs. 10 and 11, respectively.

\section{ACKNOWLEDGMENTS}

The authors ar: indebted to A. Criscuolo, D. C. Ethridge, T. H. Kuckertz, and W. M. Seifert for discussions, suggestions, and direct help and to Dolores Mills and C. W. McCabe, who kindly helped in making the punched tapes for the Intel monitor and the cassette tapes for burning in the PROMs, using the Intel-Motorola converter program.

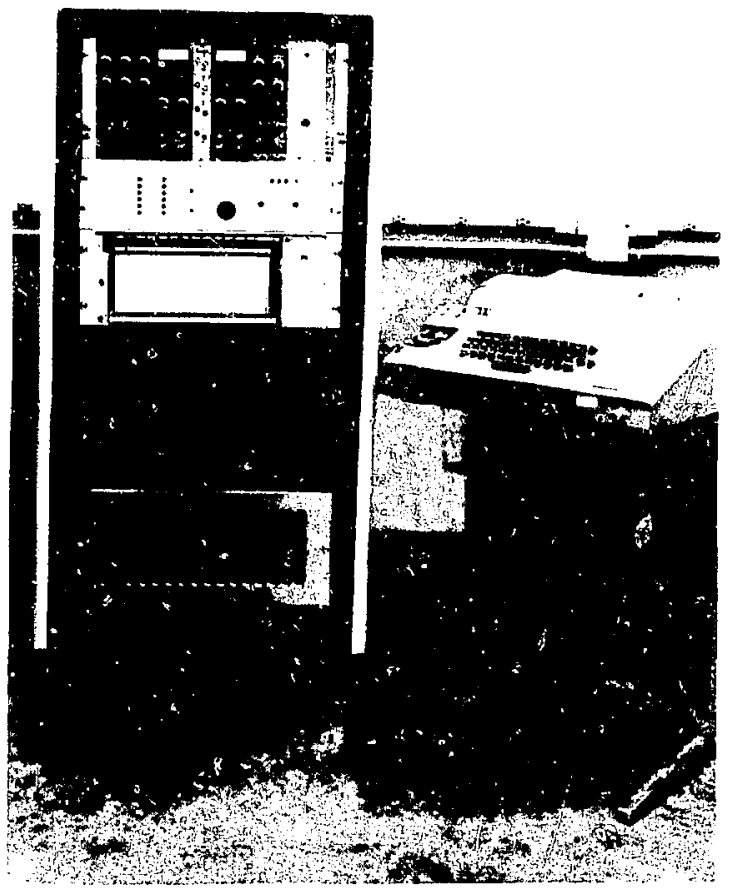

Fig. 10.

Effluent Monitor: View of the complete instrument. 

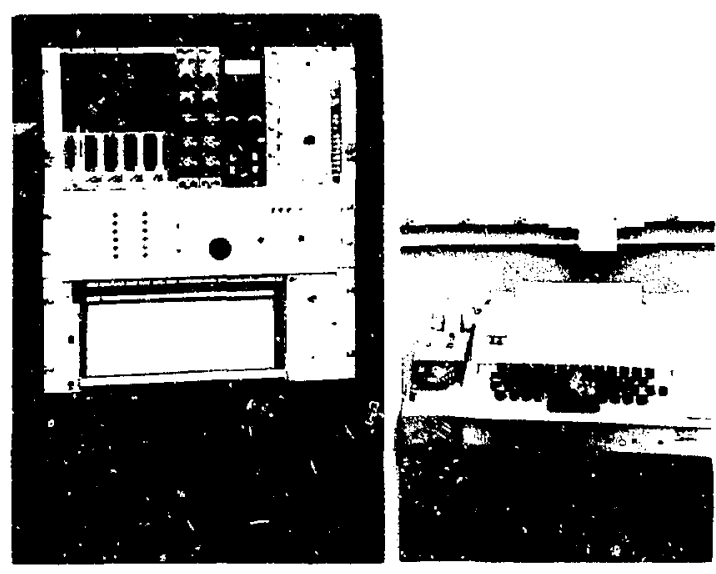

Fig. 11 .

Sanitary Seuer Monitor: View of the complete instrument.

\section{REFERENCES}

1. L. Stanchi, "Effluent Monitoring for Nuclear Safeguards," I.E.E.E. Trans. Nucl. Sci. 24, No. 1 (1977).

2. L. Stanchi, "A Self-Patching Firmware Program." Los Alamos Scientific Laboratory report LA-6634-M (December 1976).

3. L. Stanchi, "Is ASCII Code Old?" submitted for publication.

4. W. M. Seifert, "Software for the Intel 8080 Microprocessor Resident on Machine M(O)," Los Alamos Scientific Laboratory report LA-6440-MS (July 1976).

5. Intel Company, "SBC 80P Prototyping Package Users Guide," Document No. 98-223A (1976).

6. V. S. Reams, Los Alamos Scientific Laboratcry, private communication (October 1976). 